Revue Revue de l'histoire des religions

$$
\begin{array}{cl}
\text { de I histoire } & 2 \text { | } 2009 \\
\text { des religions } & \text { Varia }
\end{array}
$$

\title{
Autour d'un petit livre. Alfred Loisy cent ans après
}

Actes du colloque international tenu à Paris les 23-24 mai 2003, sous la dir. de François Laplanche, Ilaria Biagioli et Claude Langlois, Turnhout (Belgique), Brepols, 2007, 351 p., $24 \mathrm{~cm}, 47,47 €$

\section{Giacomo Losito}

\section{OpenEdition \\ Journals}

Édition électronique

URL : http://journals.openedition.org/rhr/7250

DOI : $10.4000 /$ rhr.7250

ISSN : 2105-2573

Éditeur

Armand Colin

Édition imprimée

Date de publication : 1 avril 2009

Pagination : 282-285

ISBN : 978-2200-92590-1

ISSN : 0035-1423

\section{Référence électronique}

Giacomo Losito, "Autour d'un petit livre. Alfred Loisy cent ans après ", Revue de l'histoire des religions [En ligne], 2 | 2009, mis en ligne le 12 janvier 2010, consulté le 22 septembre 2020. URL : http:// journals.openedition.org/rhr/7250 ; DOI : https://doi.org/10.4000/rhr.7250 
Autour d'un petit livre. Alfred Loisy cent ans après, Actes du colloque international tenu à Paris les 23-24 mai 2003, sous la dir. de François Laplanche, Ilaria Biagioli et Claude Langlois, Turnhout (Belgique), Brepols, 2007, 351 p., $24 \mathrm{~cm}, 47,47 €$.

Ce volume recueille les actes d'un colloque dont il faut savoir gré à Émile Goichot, Claude Langlois et François Laplanche de l'avoir organisé en mettant à contribution les meilleurs spécialistes allemands, suisses et italiens de la crise moderniste, à côté des historiens français de longue date (Pierre Colin, Bernard Montagnes et le doyen des études de Modernistica, Émile Poulat), des spécialistes des sciences religieuses de l'École Pratique des Hautes Études, des théologiens du Centre Sèvres et des chercheurs de l'Hexagone nous ayant livré les derniers grands travaux en la matière : Jérôme Grondeux, Louis-Pierre Sardella et Christian Sorrel. Ce panoptique de l'approche savante du cas Loisy - figure éponyme de la crise moderniste, dont l'œuvre « nous place devant des questions fondamentales pour la pensée catholique aussi bien que pour nos sciences humaines » (Poulat) montre bien le niveau atteint par les études en la matière; car ces dernières ont désormais dépassé l'état de la prise de parole engagée (justificatrice de la répression romaine ou prônant au contraire le procès en révision de l'affaire Loisy au Vatican), et cela quelles que soient les différentes subjectivités des spécialistes et leurs préférences personnelles. Loisy se trouve ainsi mis en perspective, au sein de la vague de rénovation des études exégétiques, historiques et anthropologiques qui, dès le XIX ${ }^{\mathrm{e}}$ siècle, déferla sur le savoir serein de l'origine, encore régnant en catholicité, malgré les secousses déjà portées par Richard Simon et par les bollandistes. La stigmatisation romaine des œuvres et de la personne de Loisy fut aussi le point culminant d'un plus vaste procès, que ce volume aide à percevoir en explorant les rapports entre l'œuvre de l'exégète français, la pensée religieuse protestante du XIX ${ }^{\mathrm{e}}$ (Laplanche) - avec laquelle il entra en une loyale compétition - et les positions de certains parmi ses contemporains. La moisson des renseignements apportés est imposante et il n'est pas possible de rendre compte ici de tous les aspects traités dans ce volume; nous ne pourrons que proposer un point de vue particulier sur le cas Loisy, suscité par la richesse du matériel recueilli, parfois inédit, comme les extraits cités par F. Laplanche du grand manuscrit conservé à la Bibliothèque nationale de France, dont Loisy tirera les articles signés Firmin et son livre L'Évangile et l'Église, parfois plus radical dans ses conclusions que sa source manuscrite.

Assurément, notre temps n'est plus celui des «cendres encore brûlantes », voire du silence des bouches cousues constaté par É. Poulat au début de son travail de pionnier. C'est que l'État laïque avait eu tendance à réduire l'affaire à une banale « querelle de curés », tandis qu'à l'opposé l'Église romaine ne voulait la considérer que comme «modeste rhume des foins » du catholicisme contemporain. Or le lecteur de cet ouvrage sera vite convaincu qu'il s'agit bien de l'une des crises majeures de notre histoire. 
En effet, pour comprendre la valeur historique de l'athéisme méthodologique, résultant de l'affirmation de l'autonomie des sciences religieuses vis-à-vis de la théologie, qui fut la devise épistémologique de Loisy dans ses travaux, il suffira de constater l'admiration soulevée par cette attitude en Allemagne, même auprès des savants protestants ses contemporains les plus importants (Troeltsch), attestée aussi par la Modernismusforschung et, dans ce volume, par un de ses représentants les plus éminents : Otto Weiss. Par ailleurs, l'importance de l'œuvre de Loisy pour le lent travail de mise à jour des sciences religieuses en Italie a été documentée par Rocco Cerrato, grâce à sa reconstruction des difficiles relations entre Loisy et l'un des chefs de file du modernisme italien, Ernesto Buonaiuti, qui joua un grand rôle dans le développement des sciences religieuses de la Péninsule. Même dans le contexte catholique hispanique, pourtant assez réfractaire, Alfonso Botti nous rappelle que L'Evangile et l'Église fut le seul texte de la mouvance moderniste (avec le roman de l'Italien Fogazzaro Il Santo) à avoir mérité une traduction (1910) et que le cardinal Vivès y Tutò intervint publiquement dans le but d'immuniser l'opinion catholique contre la dangereuse influence de Loisy.

Cela n'étonne donc pas que l'affaire engageât à Rome des personnalités de différentes nationalités, comme le montre un autre spécialiste allemand, Claus Arnold, auquel le public francophone doit aussi de pouvoir découvrir les complexes manœuvres dont les œuvres de Loisy firent l'objet aux congrégations de l'Index et du Saint-Office, où tout le monde ne manifestait pas cependant le même esprit étroit (voir les cas de Fleming, Lepidi et surtout Gismondi). Assurément, comme le montre Francesco Beretta, longtemps l'élite faisant l'orthodoxie en catholicité ne fut pas en mesure de comprendre la nécessité de faire place dans sa boîte à outils intellectuels aux exigences manifestées, sinon par Loisy, au moins par les représentants de l'exégèse, de l'histoire et de la pensée philosophique catholiques progressistes. À ce propos, les relations, souvent critiques, entretenues avec Loisy et son œuvre par Lagrange, Mgr Mignot, le lazariste Pouget, le jésuite Prat, Goyau et Blondel ont été à la fois documentées et analysées par B. Montagnes, L.-P. Sardella, Erminio Antonello, Pierre Gibert, J. Grondeux et Rosanna Ciappa. À eux le mérite de n'avoir pas non plus manqué, à l'occasion, de signaler les thèses de Loisy apparaissant encore aujourd'hui plus plausibles que celles de ses critiques catholiques à l'esprit ouvert. C'est notamment le cas de l'affirmation du caractère stratifié du livre des Actes des Apôtres, qu'en 1923 le P. Prat se refusait d'admettre et qui est désormais un présupposé acquis pour tous les exégètes contemporains, ou du refus de Loisy de subordonner l'histoire critique à l'histoire sacrée, comme le prônait Blondel dans sa conception de la tradition vivante (même si, à ce sujet, il y a peut-être encore quelques savants, y compris parmi les collaborateurs du présent volume, qui ne cautionneraient pas ce jugement).

Une évaluation également documentée et équilibrée de l'actualité des études de Loisy en matière d'exégèse néo-testamentaire, de méthode historique (fusionnant, fût-ce d'une façon encore très rudimentaire, l'exégèse 
textuelle et les résultats des sciences auxiliaires), d'histoire du judaïsme et des origines chrétiennes se trouve dans les contributions respectives de Frédéric Amsler, Arnaud Sérandour, André Lemaire et Simon Mimouni.

Ce dernier a justement remarqué la difficulté pour Loisy de prendre la mesure du rôle joué par Paul dans l'histoire du christianisme et sa sousévaluation du judaïsme chrétien, que les découvertes successives auront très probablement aidé à reconsidérer. Il nous paraît toutefois excessif de faire grief à Loisy d'un préjugé anti-judaïque : les deux " petits livres » rouges de Loisy, insistant sur le caractère juif de la mentalité de Jésus, se démarquent des passions soulevées au même moment (1902 et 1903) par l'affaire Dreyfus, à une époque où Charles Maurras exaltait un catholicisme païen et anti-chrétien (à savoir... anti-juif); Loisy offrit en outre une lecture originale et positive du judaïsme sacerdotal d'après l'exil babylonien (Sérandour). Mais il est clair, comme l'expliquent d'autres contributions (Laplanche, Koch-Piettre), que le Nazaréen et le christianisme représentèrent toujours pour lui le tournant le plus important du procès de l'histoire spirituelle de l'humanité qu'il crut découvrir à travers son étude des faits, poursuivi à la loupe d'une pensée influencée par la philosophie de l'histoire des idéalistes allemands. Christoph Theobald souligne à juste titre en conclusion de sa riche étude de l'œuvre « catholique » de Loisy, à la lumière des développements de la théologie de la Révélation de Vatican I à aujourd'hui, que l'exégète et historien français « a perçu et tenté de penser pendant sa période catholique le scandale de la singularité chrétienne, inscrit dans la chair de l'histoire. " Renée Koch-Piettre et P. Colin nous livrent le sens de l'œuvre de Loisy après sa sortie de catholicité, lorsqu'il devint l'historien des religions du Collège de France, ou mieux, un historien de la vraie religion, c'est-à-dire la morale, une religion libérée de l'orthodoxie de la croyance; cela fut une attitude appréciée par son collègue belge Franz Cumont, mais aussi par des modernistes, également excommuniés, mais demeurés plus soucieux que Loisy de la destinée du christianisme, reconnaissant encore dans l'historien français leur « maître » (Buonaiuti) ou capables d'affirmer que même « son œuvre tardive exprime le type le plus profond de la prêtrise catholique », comme le fit Miss Petre (v. les conclusions de Biagioli).

Certainement la perspective de Loisy historien des religions nous plonge en plein $\mathrm{XIX}^{\mathrm{e}}$ siècle et n'est plus praticable par les sciences religieuses contemporaines, bien qu'une théologie vraiment soucieuse de sa vocation œcuménique puisse peut-être encore en profiter. Ce sont là des données qui cadrent parfaitement avec la thèse de F. Laplanche saisissant le «néo-catholicisme " comme un caractère fondamental de l'œuvre de Loisy. Car, contre l'individualisme des protestants libéraux, il refusait d'opposer la valeur de l'Écriture, telle qu'elle s'offre à la lecture du sujet, aux acquis cumulés par la Tradition; tout en insistant - au grand scandale des tenants de l'orthodoxie catholique sous Pie X - sur l'ineffabilité du rapport entre l'homme et Dieu, dont les différents symboles religieux n'arrivent jamais à épuiser l'infinie profondeur. Pourtant, si ce type de théisme agnostique rend finalement irrecevable le modèle d'histoire des 
religions pratiqué par Loisy, en en compromettant l'athéisme de méthode, Laplanche fait justement remarquer que du point de vue de la conscience épistémologique Loisy ne fut pas aussi naïf qu'on a pu le croire; car, ne serait-ce qu'à un degré encore embryonnaire, il avait conscience du poids de la subjectivité intervenant dans le travail de compréhension de l'historien.

$\mathrm{Si}$, comme le montrent les contributions déjà citées de R. Koch-Piettre et $\mathrm{P}$. Colin, l'expérience du carnage de la Première Guerre mondiale fut un tournant autant et voire plus marquant que l'excommunication pour l'évolution de la pensée de Loisy, il ne nous reste qu'à souhaiter la parution du travail que lui a consacré Elisabeth Scheele; la synthèse complète devrait en être accessible, ainsi que celle des autres contributions au colloque organisé en 2003 par la Société internationale d'études sur Alfred Loisy, sur le site Internet de cette dernière. Le centenaire de l'excommunication devrait être l'occasion de nouvelles parutions : les actes du dossier romain de la mise à l'Index de Loisy, en 1903, préparant le décret Lamentabili, en 1907; ainsi que la publication du grand manuscrit de Loisy conservé à la $\mathrm{BnF}$, signalé plus haut.

Enfin le volume indique quelques pistes que les chercheurs seront bien inspirés d'explorer : les contributions de F. Laplanche, E. Antonello et A. Botti indiquent la nécessité d'une étude sur les Annales de philosophie chrétienne, une revue qui fut autour de 1900 une importante tribune française du renouveau de la pensée catholique. De nouvelles mises au point s'imposent également sur les relations entre Loisy et von Hügel, comme entre Loisy et Desjardins (surtout après la publication des actes du colloque organisé en 2006 par François Chaubet sur Pontigny et le modernisme). Il convient de rappeler combien de sources considérables pour les historiens de la crise moderniste (et de son long sillage de ruines) attendent de pouvoir être à plein exploitées. Pour n'en citer qu'une, le grand Fonds Laberthonnière, en libre consultation dès 2007, mais nécessitant encore un classement rigoureux et cela même en dépit de plusieurs appels à chercheurs, généreusement répétés par la $\mathrm{BnF}$.

Giacomo Losito, Université J. W. Gœethe, Francfort-sur-le-Main.

Massignon - Abd-el-Jalil : parrain et filleul, 1926-1962. Correspondance rassemblée et annotée par Françoise JACQUIN; préface par Maurice Borrmans. Paris, Le Cerf, 2007, 298 p., pl., 24 cm (« Histoire »).

L'action, les écrits et la personnalité de Louis Massignon ont dès son vivant largement dépassé le cadre des études arabes et islamiques. Militant humaniste, intellectuel catholique engagé, il a en effet exercé une influence profonde sur nombre de ses contemporains. Celle-ci a déjà été l'objet de nombreuses études consacrées à la biographie de Massignon (notamment : Guy Harpigny, 1981; Vincent Monteil, 1987; Christian Destremeau et 